\title{
Vieja historia del cannabis y recientes prácticas preventivas en Europa
}

\author{
Merino, P.P. \\ European Monitoring Centre for drugs and drug addiction \\ Administradora científica. \\ Correspondencia a: Petra Paula Merino EMCDDA. Rua Cruz de Santa Apolonia, 23. \\ 1100 Lisboa (Portugal) e-mail: Petra.Paula.Merino@emcdda.org
}

\section{Resumen}

Se analiza las informaciones facilitadas por los puntos focales del European Monitoring Centre for drugs and drug addiction (EMCDDA) en cada uno de los estados miembros de la UE. La información hace referencia a los desarrollos recientes en los programas de prevención del uso de cannabis. Incluye también una reflexión histórica acerca de los usos de esta substancia. El texto señala que en el ámbito del cannabis la prevención no es el debate principal en Europa. Este es también el caso para el tratamiento del consumo de cannabis. Llama la atención sobre el impulso que las medidas alternativas a la prisión para los consumidores y poseedores de cannabis están tomando en los estados miembros de la Unión Europea.

Palabras clave: Prevención, tratamiento, cannabis, delta9-tetrahydro cannabinol, Europa.

\section{EVOLUCIÓN HISTÓRICA}

as modas pueden hacer refrescar afinidades estéticas que se manifiestan en diferentes consumos, por supuesto que no hablo solo del de drogas, todos ellos girando en torno a un deseable y mutante estilo de vida. Los gustos por viejas usanzas se hacen eco generalmente de modas con las que las recientes generaciones ya han convivido, así que a los padres les resulta familiar lo que sus hijos comienzan a descubrir. Unos y otros puede que desconozcan lo viejas, pero viejas, que las modas son. Si en alguna

\section{Abstract}

The paper analyses the information provided to the EMCDDA by Reitox Focal Points. The information relates to recent developments in drugs prevention programmes for cannabis consumption. It also includes an historical perspective on the use of the substance. The text points out that in the cannabis arena prevention is not the principal debate in Europe. This is also the case for the treatment of cannabis consumption. The paper highlights a common trend in the context of the criminal justice systems. This trend is an increase in alternative measures to prison for cannabis consumers in the EU Member states.

Key words: Prevention, treatment, cannabis, delta9tetrahydro cannabinol, Europe.

droga tiene sentido hablar de la perspectiva histórica esta es sin duda el cannabis. No solo las utilidades remotas que se le atribuyen, sino la variedad de ellas, y su popularidad a lo ancho y largo del Planeta, hacen de él un objeto cuanto menos " curioso ".

Los usos textiles, médicos y eufóricos del cannabis se remontan a la era anterior a la nuestra. Planta usada en la antigüedad, los expertos antropólogos le calculan una edad de 6000 años, pasó a formar parte del repertorio de remedios de los ya muy avanzados médicos chinos, quienes la usaban en el alivio de enfermedades tan comunes como la 
malaria o el reumatismo. La memoria musical, tan relevante en la reciente historia de las drogas, y me refiero a la historia de las generaciones con las que nos ha tocado convivir, nos trae a la memoria el uso que del cannabis se hacia en los años 50 en la escena de jazz europea. En los años 60 y 70, el uso del cannabis como substancia euforizante se desarrolla paralelamente a la cultura hippie. Su consumo pasó a estabilizarse en algunos países hasta su resurgimiento en los finales de los 80 en un amplio espectro geográfico y social. En Europa el uso del cannabis se extiende como nunca anteriormente.

Esta extensión en el uso responde al efecto acumulativo de poblaciones de individuos que iniciaron hace varios años su consumo, dónde parece que su evolución se caracteriza por largos períodos de abstinencia total, aceleraciones y enlentecimientos. Este grupo de "viejos" consumidores sería heterogéneo. No obstante del incremento reciente en su consumo podríamos aproximarnos a un fenómeno de subcultura perteneciente a las culturas jóvenes. Las subculturas, estando en los márgenes de la industria cultural al otro extremo de la denominada industria del ocio, generarían la creación de oportunidades de trabajo, como parte de un sector de la economía marginal, indocumentada por definición. La compra, venta y producción de cannabis en este contexto no tendrían lugar en el vacío, sino que estarían íntimamente conectadas a un estilo de vida. Este es pues otro de los aspectos a tener en cuenta en las políticas preventivas.

\section{DEBATE SOBRE EL CANNABIS EN LA UE}

El Observatorio Europea de las Drogodependencias (EMCDDA) ha dedicado este año un capítulo especial al cannabis. La actualidad del tema no está en relación con el hecho de que el cannabis sea la droga ilícita mas común en Europa (1), que es cierto, tampoco con la novedad de la substancia, que como ha quedado claro no lo es. En los debates abiertos, el interés se sitúa en su estatuto legal y en los usos terapéuticos que se le atribuyen. Hablar de prevención en relación al cannabis no es hablar de los aspectos más debatidos. El más manido es sin duda el tratamiento legal de esta substancia. Este da lugar a un colorido mapa Europeo del cannabis. En efecto, se da una amplia gradación en el tratamiento legal de la posesión y consumo y cannabis, desde la tolerancia, hasta la aplicación de sanciones administrativas o penales más o menos severas.

En cuanto a los usos médicos en la Unión Europea, Alemania es el único país que ha avanzado en la dirección de permitir el uso de cannabis para tales fines. Efectivamente, en 1988 un cambio en la ley de narcóticos dió paso a el permiso de usar THC con fines terapéuticos. En Holanda se considera la posibilidad por parte del gobierno de crear una agencia que controle el cultivo y el procesamiento del cannabis con fines científicos. En UK un subcomité de la Cámara de los Lores lanzó una investigación en 1997 acerca del uso del cannabis con fines médicos y reacreacionales. El sucomité lanzo su informe en noviembre de 1998 concluyendo que existía suficiente evidencia clínica y anecdótica acerca del valor médico del cannabis y recomendó que los médicos podrían recetarlo a fin de aliviar el dolor y los síntomas de la esclerosis múltiple. En los que se refiere al uso recreacional, el subcomité no se mostró favorable a reprimir su uso. El gobierno rechazó las recomendaciones basándose en la falta de ensayos clínicos. Un nuevo estudio clínico comenzó en 1999, los resultados se esperan para 2004.

\section{CARACTERÍSTICAS DE LOS CONSUMI- DORES EUROPEOS}

Se tiene constancia de varios tipos de uso diferenciado, no obstante el mas común se aproximaría a este patrón: consumo rutinario resultado de un cierto aprendizaje del producto y de sus efectos. Dentro de la categoría 
general de consumidores de substancias ilícitas, el grupo de consumidores de cannabis se caracteriza diferencialmente de aquel que constituido por los que solicitan asistencia o de los que entran en prisión. La norma es la integración social, diversificada eso sí, caracterizada por la ausencia de estigmas severos de carácter médico - legal y la rareza de situaciones adictivas. Esta descripción nos sitúa frente al hecho de que en la mayor parte de la Union Europea el cannabis no se asocie a un contexto social o recreacional exclusivamente. Las percepción más generalizada en la población tiende a considerar el consumo del cannabis como una acto mundano y no desviado. El cannabis no ha despertado lo que la autora británica Angela Mcrobbie denomina "pánico moral", como lo habría provocado el éxtasis a través de los medios de comunicación social. El consumo de cannabis se sitúa en la esfera privada, lo que hace que no sean el vínculo que podría hacer aproximarse a grupos muy diferenciados socialmente, como es le caso de la heroína, por ejemplo, en el que el consumo callejero. En Holanda, Cohen y Sas (1997), el cannabis generalmente se encuentra en la historia de consumo precediendo el uso de otras drogas. No obstante, la gran mayoría de personas que han usado alguna vez cannabis nunca han experimentado con otras drogas.

\section{RIESGOS DEL CONSUMO DE CANNA- BIS}

El consumo de cannabis ha sido escasamente descrito como aquel que dará lugar a problemas de salud. Cuando tales consecuencias son evocadas, se trata generalmente de problemas que siguen inmediatamente a un consumo preciso.

Según Ingold y Toussirt (6), como riesgos se han señalado la posibilidad de descompensación de enfermedades psiquiátricas y la existencia de conductas de dependencia. Las segundas afectarían sobre todo a jóvenes en situación de sufrimiento social o psicológico.
El sujeto se concentraría sobre actividades que giran obsesivamente en torno al cannabis, consumo frecuente, rodearse sólo de otros consumidores, reventa. Estos consumos dejan de ser recreativos y pasan a ser de ser un objeto de investimiento total, el único lugar social que une al individuo a los otros.

En relación a la asociación, causal o no, entre cannabis y patologías mentales existirían según Hall (3) dos hipótesis centrales. La primera sería que un uso repetitivo de cannabis puede causar la denominada "psicosis de cannabis" - psicosis que nunca aparecería sin el uso de la substancia, los síntomas estarían precedidos por un intenso uso del cannabis y remitirían cuando se produce la abstinencia. La segunda hipótesis es que el uso de cannabis puede precipitar la esquizofrenia, o exacerbar sus síntomas. Parece existir algún apoyo clínico en relación a la primera hipótesis. Si bien estos desórdenes existen parecen ser no habituales, pues requieren altas dosis de THC, el uso prolongado de formas de cannabis altamente potente, o una cierta vulnerabilidad. Existe mayor apoyo a la segunda hipótesis. Un amplio estudio prospectivo ha mostrado una relación linear entre la frecuencia con que el cannabis ha sido usado por jóvenes de 18 anos y los riesgos que a lo largo de los 15 anos siguientes una esquizofrenia pueda ser diagnosticada. Sigue siendo poco claro en que momento esto significa que el uso de cannabis precipitaría la esquizofrenia o es una forma de auto-medicacion, o si la asociación se debe al uso de otras drogas, tales como las anfetaminas, que con frecuencia son consumidas por consumidores intensos de cannabis. Existe una evidencia más clara de que el cannabis pueda exacerbar los síntomas de la esquizofrenia. Una implicación clara para los servicios de prevención que puede desprenderse de las anteriores constataciones es que los servicios de salud mental deberían ser capaces de identificar pacientes con esquizofrenia que usan alcohol, cannabis y otras drogas y recordarles que se abstengan o que reduzcan con- 


\begin{tabular}{|l|c|c|c|c|}
\hline \multicolumn{5}{|c|}{$\begin{array}{c}\text { Comparación de los efectos adversos sobre la salud en los usuarios habituales } \\
\text { de las sustancias más frecuentes }\end{array}$} \\
\hline & MARIHUANA & ALCOHOL & TABACO & HEROÍNA \\
\hline Accidentes de trafico y otros & $X$ & $X X$ & & $X$ \\
Violencia y suicidio & & $X X$ & & $X X$ \\
Muerte por sobredosis & & $X$ & & $X X$ \\
HIV & & $X X$ & & \\
Cirrosis & $X X$ & $X X$ & \\
Infarto & $X$ & $X$ & $X X$ & \\
Enfermedades respiratorias & $X$ & $X$ & $X X$ \\
Cánceres & $X X$ & $X X$ & $X$ \\
Enfermedades mentales & $X$ & $X X$ & $X$ & $X$ \\
Dependencia/adición & $X X$ & \\
Efectos en el feto & $X X$ & \\
\hline \\
(fuente: Wayne Hall, Robin Room y Susan Bondy, Comparing the health and psychological risk of alcohol, cannabis, nicotine and opiate use) \\
\hline
\end{tabular}

siderablemente su uso de substancias psico activas.

De acuerdo con Hall y otros (5), desde la perspectiva del individuo los problemas mas intensos de salud se asociarían al uso diario y continuado a lo largo de los años. La prevalencia de tales problemas correspondería a la secuencia decreciente siguiente: desarrollo de síndrome de dependencia, desarrollo de bronquitis crónica, implicación en un accidente de trafico bajo los efectos de las substancia. En cualquiera de estos casos el riesgo se vería aumentado si el cannabis se combina con alcohol o tabaco, o ambos. El riesgo mas probable para un usuario ocasional sería el de un accidente de carretera, especialmente si su ingestión se combina con el alcohol.

\section{DEMANDAS DE ASISTENCIAY SUS IM- PLICACIONES PARA LA PREVENCIÓN}

El recurso a los servicios de salud ligados a al consumo de cannabis en la mayoría de los países de la UE son raros. Se produce generalmente en el cuadro de intervenciones familiares o en aquellas ligadas al medio escolástico. Pueden asimismo tener lugar en el marco de una decisión judicial, a través de las medidas de asistencia terapéutica ordenadas por un juez. Otra circunstancia que se encuentra es la de un consumo de cannabis que viene a enmascarar, o poner en evidencia, una patología mental o el consumo de otros productos. En general los usuarios de cannabis no desarrollan problemas relevantes que les hagan acudir a los servicios. No obstante en los últimos anos se ha producido un aumento en el numero de clientes que acuden a tales servicios. La mayoría son jóvenes, algunos de ellos con múltiples consumos, generalmente éxtasis y otras drogas, y cuya droga primaria referida es el cannabis. No obstante el cannabis es registrado de manera mas común como droga secundaria en aquellas personas que entran en tratamiento.

EI OEDT no dispone de información detallada acerca de las características de los usuarios de los servicios de apoyo a jóvenes o de aquellos enmarcados en la asistencia sanitaria primaria. En su mayoría los datos corresponden a los centros de tratamiento especializados. A fin de diseñar medidas preventivas adecuadas sería aconsejable una mejor comprensión acerca de la extensión y características de los problemas relacionados con el cannabis y de un dibujo más claro acerca de la demanda de tratamiento. En relación a esto, estas serían algunas de las preguntas que el OEDT propone para el desarrollo de medidas de prevención en el ámbito de la UE: ¿Los problemas son causados principalmente por el cannabis, o existen otras drogas implicadas incluyendo el alcohol?, ¿es el can- 
nabis una etiqueta aconsejable para englobar un conjunto de patologías de salud mental, incluyendo los suicidios, que están creciendo entre adolescentes y jóvenes?, ¿hasta que punto el aumento de la demanda de tratamiento refleja un aumento en la prevalencia, frecuencia de uso y la potencia de la substancia?, ¿se han producido cambios en los servicios orientados a la prevención secundaria que puedan acoger este tipo de fenómenos? ¿ha afectado a los usuarios de cannabis la aplicación de medidas terapéuticas o medidas alternativas a la prosecución y a la prisión?

\section{CARACTERÍSTICAS DE LAS PRÁCTICAS PREVENTIVAS EN RELACIÓN AL CAN- NABIS EN LA UE}

Las prácticas preventivas no reflejan un tratamiento especial para el cannabis. En efecto, de los programas de prevención identificados por el Observatorio Europeo tan sólo una escasa minoría van dirigidos exclusivamente a la prevención del uso del cannabis. En su mayoría se dirigen a prevenir el uso excesivo y sólo en última instancia persiguen la abstinencia total. Los materiales informativos que se editan incluyendo las drogas más usadas suelen incluir el cannabis. La modalidad informativa que constituyen las líneas de apoyo telefónico también ofrecen asistencia en relación al cannabis. No se ha encontrado, no obstante, ninguna de tales líneas que se dedique en exclusiva a los usuarios del cannabis. Los profesionales debaten la pertinencia o no de crear programas específicos, la misma cuestión se plantea en los servicios de tratamiento donde se considera la posibilidad de ofrecer asistencia en el marco de otras intervenciones no específicas.

En general, la política de drogas en la Unión Europea puede considerarse un balance entre la represión y la tolerancia. Por un lado se intentan controlar los efectos dañinos del consumo de drogas, y por otro se trata de controlar la oferta y disponibilidad de drogas a través de medidas legales y represivas, si bien la introducción de medidas alternativas a la represión es una práctica que se ha venido desarrollando en los últimos tiempos. En efecto, las medidas alternativas a la prisión en casos de posesión y consumo de cannabis, es una de las tendencias comunes más claramente marcadas en los estados miembros de la Unión Europea. Este hecho se produce paralelamente al incremento de arrestos policiales en relación a la posesión y consumo de cannabis. En este contexto conviene citar que el cannabis puede ser detectado en la orina dos semanas después de su uso, incluso durante un periodo mas largo en el caso de consumidores crónicos. Este periodo es considerablemente mayor para el cannabis que para otras drogas, dónde generalmente el tiempo de detección en orina es de uno a tres días. El Observatorio Europeo aún no ha analizado suficientemente como la política de drogas se aplica en la práctica en relación al cannabis, para ello se necesitarían llevar a cabo estudios que analizaran datos referidos a la policía y al sistema judicial más en detalle. Teniendo en cuenta que la entrada por vez primera en la prisión aumenta para un individuo considerablemente las posibilidades de iniciar el circulo prisión-libertad-prisión, estas medidas pueden considerarse benéficas en términos preventivos globales y con un impacto positivo en la salud pública.

\section{ALGUNOS PROGRAMAS DE PREVEN- CIÓN DEL CONSUMO DE CANNABIS Y RIESGOS ASOCIADOS EN LA UE}

El informe annual del OEDT de 1999 hace referencia a varios programas de prevención. Los citamos aquí de manera yuxtapuesta por la utilidad que para algún lector pueda suponer la identificación de prácticas sobre el terreno.

En la Comunidad flamenca de Bélgica fue creado en 1997 un grupo de autayuda para consumidores de cannabis. 
En 1997 en Dinamarca se editó material escolar para jóvenes escolarizados entre 13 y 16 anos. Dicho material incluye folletos informativos, libros, casetes de vídeo para quienes desean conocer más acerca de la droga o para aquellos que experimentan problemas con ella. Uno de los folletos informativos está destinado a los padres y resuelve dudas acerca de las características de esta substancia.

En Luxemburgo se han organizado una serie de actividades formativas, entre las que se encuentra un seminario sobre el cannabis destinado trabajadores sociales y otros agentes en el campo de las drogas.

En Holanda, las actividades de prevención se han dirigido a grupos específicos a riesgo y se han centrado en el cannabis. Dos campañas mas media fueron lanzadas en 1996 y 1997, una dirigida a los padres y la otra a los jóvenes. El mensaje central en la campaña a los padres fue infórmese usted mismo, y fue diseminada en un amplio sector de medios de comunicación. La campaña fue reestructurada para los jóvenes al año siguiente y se conectó con otras actividades a nivel local y regional.

En España se han lanzado programas de prevención en escuelas dirigidos a jóvenes de 12 a 16 anos, implicando, ONGs y complementadas con campañas a nivel nacional en las que se usaron folletos, anuncios televisados y carteles. Los programas de formación fueron ofrecidos a profesores, voluntarios de
ONGs y otros grupos profesionales a fin de poder trabajar en el campo del cannabis.

Durante algunos años, en Suecia se ha venido publicando el Libro del hashish, que ha siso distribuido a los padres de adolescentes. En 1998, fue reemplazado por el libro The book of drugs, que cubre todas las drogas incluyendo alcohol y tabaco.

\section{BIBLIOGRAFIA}

1. Amador Calafat, Karl Bohrn, Montserrat Juan et al. Night life in Europe and recreative drug use. SONAR 98. Irefrea \& European Commission. Palma de Mallorca 1999.

2. Extended annual report on the state of the drugs problem in the European Union. EMCDDA. Lisbon. 1999.

3. Wayne Hall. Cannabis Use and Psychosis. National Drug and Alcohol Research Centre. Sydney peper presented at: Problematic Alcohol \& Drug Use \& Mental Illness Melbourne, February 1998.ISBN: 0947229884 Copyright NDARC 1988

4. Angela Mcrobbie. Postmodernism and popular culture. Routledge. London. 1998.

5. Harold Kalant, Willian A Corrigal, Wayne Hall et al. The Health effect of cannabis. The addiction resaerch foundation. Toronto. 1999

6. Rodolphe Ingold, Mohamed Toussirt. Le cannabis en France. Paris. Anthropos. 1998.

7. Kleanthis Grivas. Cannabis: marihuana - hashish. Minerva press. London. 1997. 